\title{
CoreView: Fresh tissue biopsy assessment at the bedside using a millifluidic imaging chip
}

\author{
David J. Cooper, ${ }^{* a}$ Chuqin Huang, ${ }^{b}$ Dylan A. Klavins, ${ }^{c}$ Mark E. Fauver, ${ }^{c}$ Matthew D. Carson, ${ }^{c}$ Farzad Fereidouni, ${ }^{\text {d Suzanne }}$ \\ Dintzis, ${ }^{e}$ Csaba Galambos ${ }^{f}$, and Eric J. Seibel*c

\begin{abstract}
Minimally invasive core needle biopsies for medical diagnoses have become increasingly common for many diseases. Although tis sue cores can yield more diagnostic information than fine needle biopsies and cytologic evaluation, there is no rapid evaluation at the point-of-care for intact tissue cores that is low-cost and non-destructive to the biopsy. We have developed a proof-of-concept 3D printed millifluidic histopathology lab-on-a-chip device to automatically handle, process, and image fresh core needle biopsies. This device, named CoreView, includes modules for bio psy removal from the acquisition tool, transport, staining and rinsing, imaging, segmentation, and multiplexed storage. Reliable removal from side-cutting needles and bidirectional fluid transport of core needle biopsies from five tissue types has been demonstrated with 0.5 -mm positioning accuracy. Automation is aided by a MATLAB-based biopsy tracking algorithm that can detect the location of tissue and air bubbles in the channels of the millifluidic chip. With current and emerging optical imaging technologies, CoreView can be used for a rapid adequacy test at the point-of-care for tissue identification and glomeruli counting from renal core needle biopsies, and has the potential to be used for breast cancer diagnosis, phenotyping, and mapping of enriched
\end{abstract} \\ tumorous regions for downstream analyses in global health applications.
}

\section{Introduction}

Histopathology and the technologies used to perform it have not changed significantly for over 75 years, with the exception of the development of immunohistochemistry in the 1980s. ${ }^{1}$ Current histopathology practices are tissue destructive, laborintensive, costly, and take many hours to days to complete. In addition, the traditional tissue preparation methods used by histopathology are not miniaturized nor optimized for needle biopsies, such as core (CNB) and fine needle biopsy (FNB), which have become the preferred biopsy technique for many organs due to their minimal invasiveness. ${ }^{2}$ Millions of needle biopsies are procured annually and there has been a lack of effort to automate and standardize the processing of the increasing number of molecular diagnostic tests used for personalized medicine and precision therapy.

Needle biopsies have a significantly higher incidence of inadequacy, or the lack of an adequate sample of tumor for diagnostic assessment, than surgical excisions. ${ }^{3}$ Inadequate biopsies frequently lead to diagnostic delay, biopsy procedure duplication, and/or inconclusive histopathologic results. As a result, rapid on-site evaluation (ROSE) adequacy analysis has become increasingly used to provide reliable diagnostic information of small needle biopsies in the timeliest fashion with the least burden to the patient, the biopsy operatory, and the hospital administration. ${ }^{2}$ Touch preparation (TP) is the most common ROSE technique used on small needle biopsies but requires a significant amount of skilled manual labor and cytopathology training. Furthermore, TP has limited utility for fibrous, cystic, and necrotic lesions. ${ }^{4}$ By physically rolling or squashing the tissue on a glass slide, diagnostic cells are dislodged from the biopsy, potentially damaging the specimen and producing artifacts that disrupt downstream pathologic evaluation.

To solve these problems and meet the additional needs identified by collaborating pathologists, we have developed a histopathology lab-on-a-chip device, named CoreView, based on a novel millifluidic system that automates the tissue handling process from needle acquisition to imaging. Preceded by modular microfluidic architectures in 2D and 3D, the millifluidic chip design is developed in modular format for the required steps of making a rapid disease diagnosis on a roughly $1-\mathrm{mm}$ diameter CNB. ${ }^{5-6}$ The millifluidic chip features sequential modules for biopsy removal from the procurement device, transport between modules, staining and rinsing, optical imaging, segmentation, storage, and removal. The CoreView system can be used with any crude or advanced optical microscope designed for thick-tissue 3D imaging. The addition of fixation and optical clearing can be included for deep tissue microscopic analysis being developed for slide-free volumetric histopathology. ${ }^{7}$ Our CoreView system is unique in the ability to rapidly and gently remove CNBs from side-cut needles. Furthermore, using custom bidirectional pulsatile flows enable precise non-destructive bidirectional transport and staining of these fresh biopsies for the future purposes of performing ROSE adequacy in seconds and slide-free histology in minutes.

\section{Experimental}




\section{Animal tissue models}

All biopsies used for testing the CoreView chip and its components were acquired from resected organs donated from recently euthanized female Yorkshire pigs. These pigs were undergoing trials for unrelated research in the UW Center for Industrial and Medical Ultrasound. Therefore, this project did not demand additional loss of life outside of other research being conducted on campus and did not require additional ethical considerations or requirements.

\section{D printing, post-processing, and assembly of the CoreView chip} and associated modules

All 3D printed parts were printed using a Form 3 (Formlabs, USA) Low Force Stereolithography printer. Most parts were printed using Standard Clear resin (Formlabs) with the exception of the biopsy segmentation elastomeric buffer, which was printed using Elastic 50A resin (Formlabs). Computer-aided design (CAD) models were developed using SolidWorks 2018 (SolidWorks Corporation, USA). All prints were made with the default 3D print parameters for each resin type and with auto-generated supports in the PreForm application (Formlabs). Prior to the removal of supports, all prints were washed in isopropyl alcohol (IPA) for six minutes to remove uncured photopolymer resin. Prior to post-curing, internal channels were flushed with IPA and then compressed air to remove any uncured resin or debris and to identify problematic blockages. If successfully cleared using compressed air, the parts were post-cured using manufacturer-recommended protocol in a FormCure (Formlabs) until fully hardened.

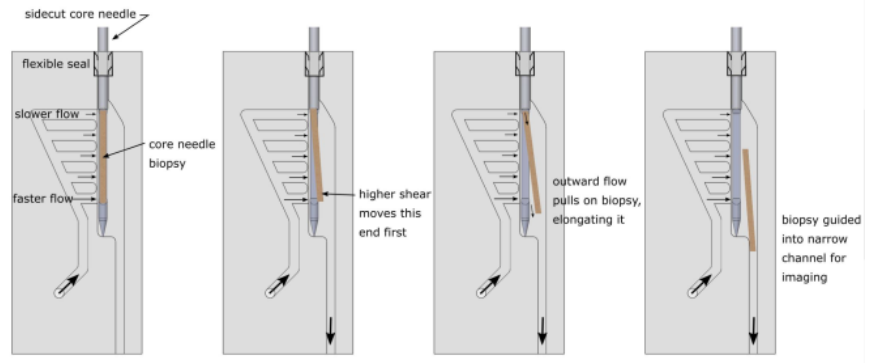

Fig. 1 Diagram of fluid mechanics involved in the removal of a CNB from a side-cut needle device using the $3 \mathrm{D}$ printed biopsy removal module. Successful removal results from the higher shear flow at the distal end of the CNB.

Aside from the channel ports on the sides of the chip, the only opening on the CoreView cartridge is the staining-and-imaging chamber. This opening is sealed using a double-sided adhesive, laser cut around the opening, to attach a desired coverslip. This prevents the resin-based chip from obscuring or distorting the histopathologic image and is the only post-print modification to the CoreView cartridge. The biopsy segmentation proof-of-concept module, however, requires significant assembly and will be described later.

\section{LabVIEW millifluidics control}

All CoreView millifluidics are controlled by a single LabVIEW 2020 (National Instruments, USA) program. This program controls the pumps and valves using dedicated custom drive electronics and a pair of data acquisition devices (USB-6002 DAQ, National Instruments). It also controls illuminations sources and captures camera image data. The current program is manually triggered to provide pulsatile flow, but the end product will be designed to automatically perform all CoreView functions.

\section{Biopsy removal module design and testing}

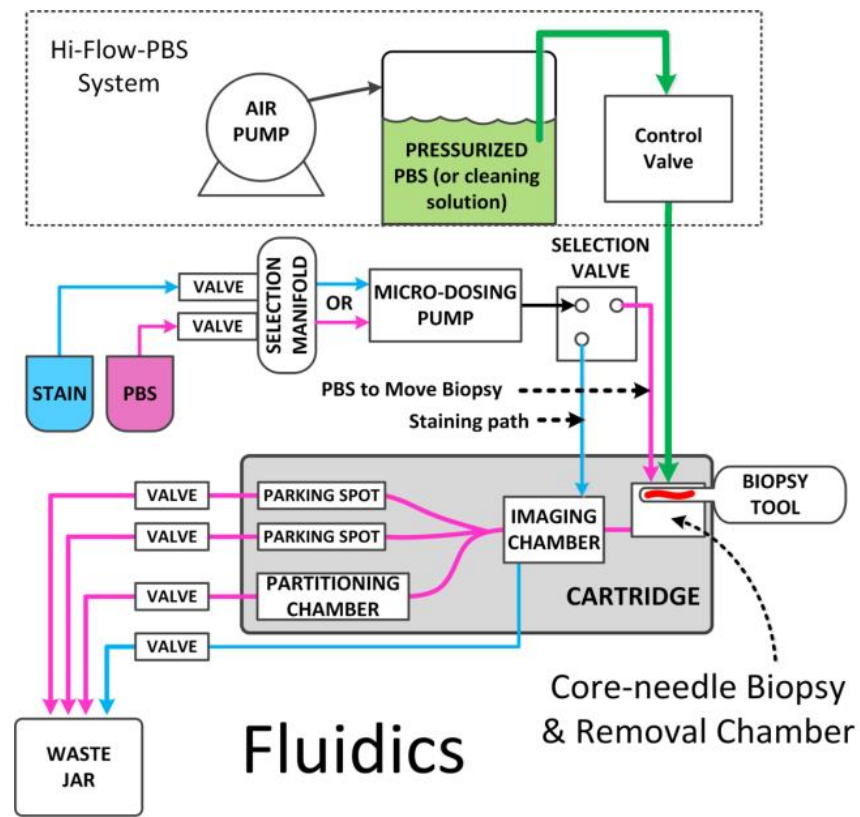

Fig. 2 Detailed diagram of the CoreView fluidics system with a simplified chip. All pumps and valves are controlled by LabVIEW with custom drive electronics and NIDAQs.

The most important feature of the CoreView histopathology on a chip is the automation because it removes the need for human handling of fresh needle biopsies. Furthermore, removing the biopsy from its needle is one of the more challenging tasks because if done incorrectly, it can distort or damage the tissue architecture. To solve these issues, we have developed a biopsy removal module that seamlessly integrates into the CoreView chip. The biopsy removal module is designed to use one source flow, divided into an array of unequal-flow-resistance channels to spatially vary the amount of fluidic shear stress along the length of the biopsy over time (Fig. 1). It distributes the volumetric flow gradient using parallel rectangular channels two times longer at the proximal end of the biopsy than at the distal end. This flow gradient, in time, is designed to be highest at the distal end of the biopsy to ensure it is separated from the needle first. This design prevents the biopsy from folding upon itself and/or breaking, which can occur if the proximal end is separated first. The pulsatile, burst flow used to remove the biopsy comes from a "Hi-flow PBS fluidic system", which provides a higher volumetric flow rate $(1-5 \mathrm{~mL} / \mathrm{s}$ in $50+\mu$ s pulses) than the transport pump (Fig. 2). This Hi-flow PBS pump is built using an air pump (KNF, Germany) and pressure regulator (SMC, USA) to pressurize a DURAN glass bottle filled with PBS (DWK Life Sciences, Shanghai). The biopsy removal module is designed to accompany most commonly used needle sizes (14-18-gauge) and ultimately moves the biopsy into the 2-mm-diameter main cylindrical channel for transport to the staining and imaging chamber. 
Proof-of-concept device. Testing of the biopsy removal module was performed in two main stages: the proof-of-concept module (Fig. 1) and the integrated chip, based on the overall conceptual design shown in Fig 3. For the proof-of-concept device, the biopsy removal module was 3D printed as its own part and connected directly to the Hi-flow PBS system of the CoreView millifluidic system. 10-mm-long 14-gauge side cut CNBs (Mission, Bard Biopsy, USA) of breast ( $N=29)$, kidney $(N=21)$, liver $(N=40)$, lung $(N=25)$, and lymph node $(N=31)$ were acquired from porcine organs that were resected, posteuthanasia. The organs used were either freshly resected (within five hours) or refrigerated for up to three days. These tissues were chosen because they are common organs for needle biopsies and each display unique mechanical characteristics that affect its ability to separate from the needle ${ }^{8-9}$. PBS was flowed in three-pulse intervals driven by a bottle pressure that was incrementally increased until the biopsy was removed. For each biopsy, we recorded the tissue type, freshness, intactness (before and after removal), successful release, and the pressure at release.

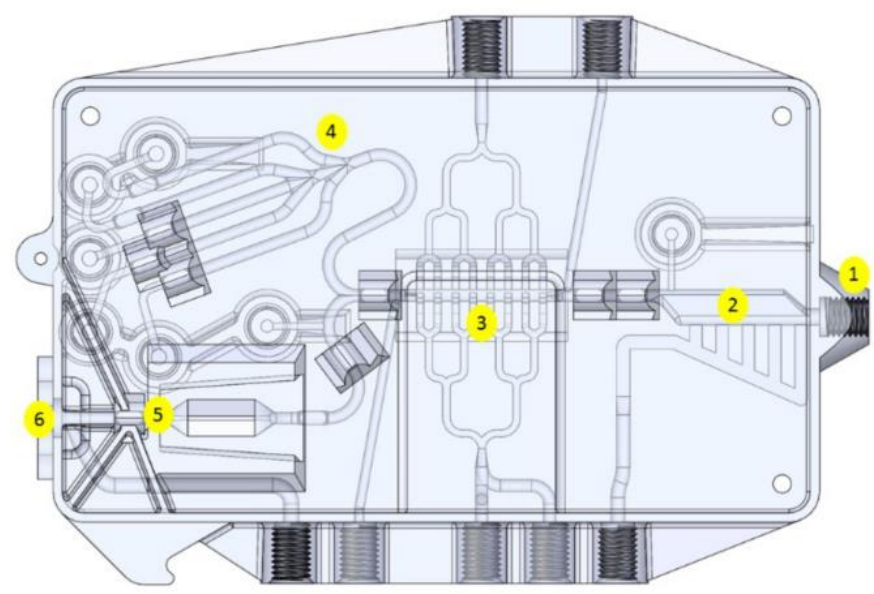

Fig. 3 CAD image of a conceptual end-product design of the CoreView chip. This model features (1) a port for needle insertion, (2) the biopsy removal module, (3) a chamber for staining and rinsing, biopsy rotation, and imaging, (4) the multiplexed storage "parking" module, (5) the biopsy segmentation module, and (6) an exit port for biopsy removal from the chip.

Biopsy removal module in the CoreView chip. To test the performance of the biopsy removal module in the integrated chip, similar methods used to test the proof-of-concept device were performed. All CNBs were acquired from freshly resected (within five hours) porcine organs (20 each of breast, kidney, lung, and pancreas) using a 14-gauge, 22-mm-long side cut needle (MaxCore, Bard Biopsy). Fresh tissue was necessary for consistency between results and relevance to CoreView clinical use, as the end product is designed to be used within seconds of resection. Additionally, to ensure the biopsy removal module works with varying needle sizes, similar testing was performed using 14, 16, and 18-gauge side cut needles (MaxCore, Bard Biopsy) and refrigerated porcine kidney.

\section{Biopsy transport and storage}

The CoreView chip relies on a single 2-mm-diameter cylindrical channel to transport the removed biopsy to and from each module. This transport is accomplished using pulsatile flow from a Type 7615 micro-dosing fluidic pump (Burkert, USA) in small, $5 \mu \mathrm{L}$, pulses with a short rise time. A short rise time is required to achieve a large $(>10)$
Womersley number, which is the main characteristic of a nonparabolic "plug flow", in Newtonian fluids. ${ }^{10-12}$ In plug flow, the velocity profile across a channel is relatively flat, allowing for the nondestructive transport of small needle biopsies, and any associated fragments, in the millifluidic channels (Fig. 4). Plug flow also keeps the biopsy in its initial elongated orientation, preventing any folding during transport.

The Womersley number is defined as:

$$
\alpha=R \sqrt{\frac{\omega \rho}{\mu}}
$$

where $\boldsymbol{R}$ is the tube radius, $\boldsymbol{\omega}$ is the angular frequency of the pulse, $\boldsymbol{\rho}$ is the fluid density, and $\boldsymbol{\mu}$ is the fluid viscosity. ${ }^{10}$ With room temperature $\left(20^{\circ} \mathrm{C}\right) \mathrm{PBS}$ and 2-mm-diameter CoreView channels, the Womersley number becomes:

$$
\alpha=0.3525 \sqrt{\omega}
$$

While this CoreView-specific Womersley number can be used to find pulse frequencies that will produce plug-like flows in clear channels, it does not account for biopsies in the chip. Qualitative experimentation with flow rates showed that plug-like flows around CNBs are achieved with lower frequencies, but require short pressure rise time during pulsation in a low-compliance fluidic system.

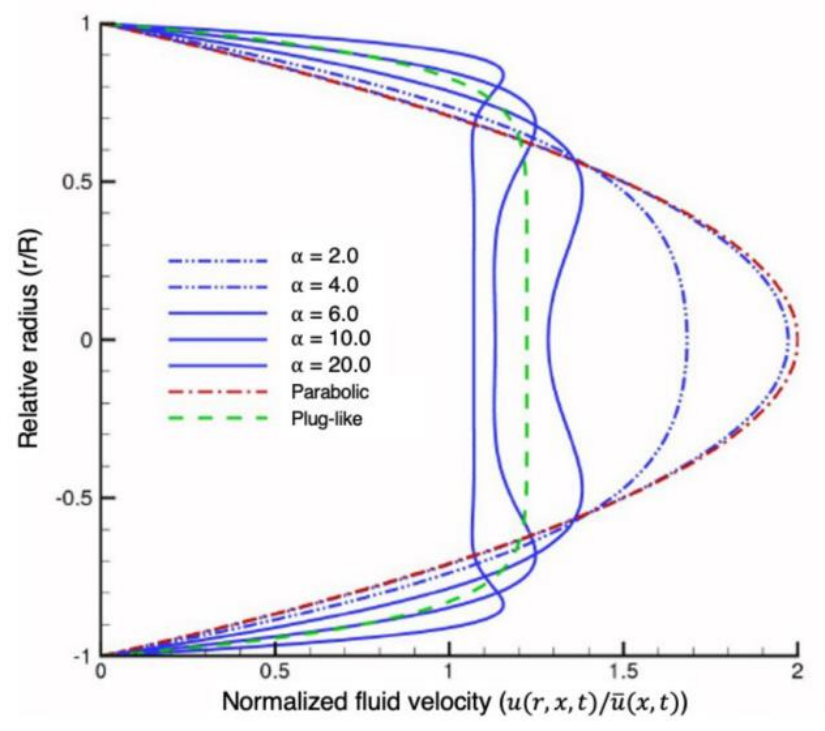

Fig. 4 Steady Hagen-Poiseuille parabolic (red) and plug-like (green) velocity profiles compared to the peak velocity profiles of the transient Womersley flow (blue). Adequate plug-like Womersley flow is achieved when $\alpha>6$. Adapted from San and Staples. ${ }^{12}$

Directed transport can be performed by using the micro-dosing pump in forward or reverse with at least one exit valve open (Fig. 2). Multiplexed storage of biopsies, referred to herein as biopsy "parking", can be achieved by opening only the desired "parking space" valve until the biopsy is in the chosen parking space channel, then closing that valve and opening another. The biopsy should not be able to leave that channel until the valve is opened again and fluid 

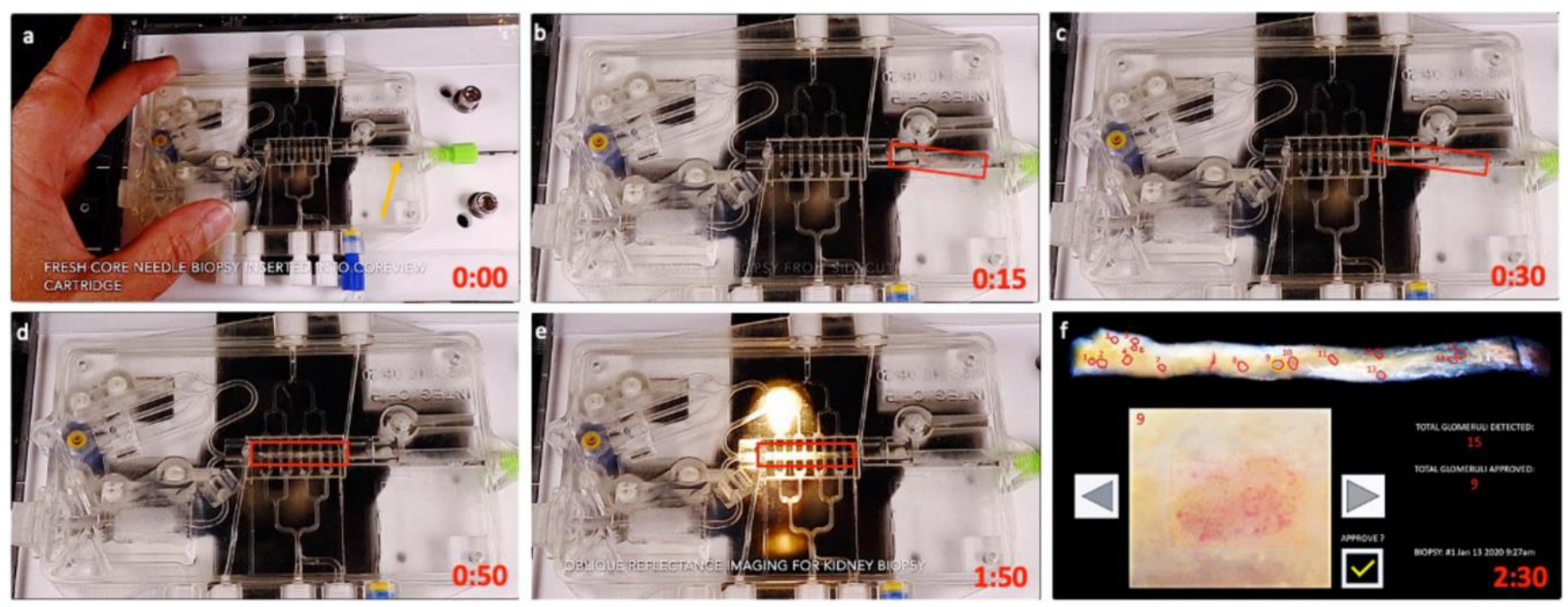

Fig 5 A sequence of video frames from Movie S1 illustrating the entire process from (a) needle insertion, (b) biopsy removal, (c) biopsy transport, (d) centering in imaging chamber with option staining, (e) imaging, and (f) detection of glomeruli in a stitched series of CNB images. The red rectangular box outlines the CNB and red numbers are minutes:seconds from needle insertion (time zero).

is driven in reverse. Biopsy parking may be ideal for in-chip fixation with formalin or other fixatives, which is required for many subsequent diagnostic procedures.

\section{Biopsy staining and imaging}

As a histopathologic device, the CoreView chip must demonstrate the ability to stain a CNB for enhanced optical image contrast. By using flow rates less than those used in biopsy transport, CNBs do not significantly move during staining and rinsing. These low flow rates do not produce a pull/push force that results in significant biopsy movement while allowing the pulsed fluid to flow around the surface of the CNB. In the CoreView chip, staining is performed in the imaging chamber using 50-75\% smaller channels for stain inflow and outflow to limit biopsy movement. After staining and subsequent rinsing, imaging is performed using optical microscopy. To image the entire length of the CNB, a computer-controlled $X-Y$ translational stage is used with optional Z-plane focus scanning for expanded depth-of-field. Full-biopsy panoramic images were mosaicked using MATLAB (MathWorks, USA) or ImageJ (NIH, USA).

With this paper, we present a video demonstration of the CoreView chip and millifluidics system (Movie S1 and Fig. 2-3, 5). This video illustrates two clinical applications: (1) ROSE adequacy of renal core biopsy that requires glomeruli counting, and (2) Detection and mapping of tumor-rich regions on the surface of a breast CNB. The demonstrated features of this chip will be discussed in the "Results and discussion" section. The video frames shown in Fig. 5, captured from Movie S1, illustrate the application of glomeruli counting using CoreView. A 20-mm-long 14-gauge porcine kidney CNB is shown undergoing insertion, removal, transport, and imaging using oblique LED illumination, a $4 x$ objective, and color camera imaging in reflection (USB 3.0 xiD CCD Ximea Corp). The final panorama of the $\mathrm{CNB}$, which is a mosaic of a series of color images, exemplifies future computer-aided detection of kidney glomeruli which enables remote pathology. The image processing for generating the panorama was conducted in MATLAB R2020b (MathWorks) in the following steps: First, a Gaussian filter was applied to each image to remove noise and a threshold was applied to remove the background data (Fig. S1a-b). Next, a contrast-limited adaptive histogram equalization (CLANE) function was applied to each channel of the Red, Green, Blue (RGB) color space and then the $S$ channel of the Hue, Saturation, Value (HSV) color space, after transferring the image, to enhance the saturation of the image (Fig. S1c-d). ${ }^{13}$ After this step, the processed images were stitched using SURF and RANSAC features and the alpha blending method. ${ }^{14-15}$ Glomeruli detection was achieved using a special weighted equation and an adaptive threshold based on regional intensity morphological operation (Fig. S1e-f).

Also included in the video is ROSE adequacy imaging of a 20-mmlong 14-gauge porcine breast CNB (Mission, Bard Biopsy) using microscopy with ultraviolet surface excitation (MUSE) imaging MUSE uses deep-ultraviolet (DUV) lasers to excite the first 10$20 \mu \mathrm{m}$ of tissue, allowing for the imaging of only the first few layers of cells. ${ }^{16}$ Prior to imaging, the breast CNB was stained in less than 1 minute with $5 \mu \mathrm{g} / \mathrm{mL}$ Hoechst 33342 (Thermo Fisher Scientific, USA) in PBS added to $10 \mu \mathrm{g} / \mathrm{mL}$ Rhodamine B (Thermo Fisher Scientific) in PBS. Hoechst is a nuclear stain that emits blue light and Rhodamine B is a cytoplasmic stain that emits yellow light when excited with 285-nm DUV light. Images were viewed and saved using Ximea CamTool (Ximea Corp.).

\section{Biopsy tracking algorithm development}

For transitioning from manual to computer control of CoreView automation, we have developed a camera-based biopsy tracking algorithm in MATLAB R2020b (MathWorks). This algorithm was developed and used on a 16G RAM PC desktop with a Ryzen 3600XT 
processor (AMD, USA) and a GeForce GTX 1660 Ti graphics card (Nvidia, USA). To develop the algorithm, 14-gauge porcine kidney CNBs were acquired and input into the CoreView chip and transported throughout the channels. The chip was backlit using a bank of white LEDs under a plastic white diffuser and videos were taken using a color video camera (MOKOSE 4K, Shenzhen Yunlang Technology Co., China). In all the following implementations of camera tracking, temporal frequency of the video camera was $10 \mathrm{~Hz}$ or greater, which limited the speed of the fluidic functions for feedback control.

Identifying the biopsy in each frame (frame tracking). The main targets of the algorithm are the biopsy and its possible fragments. Due to variable illumination, the color and saturation of the biopsy is not constant during transport. As a result, the algorithm must be insensitive to environmental changes. To create this algorithm, we first compared the mean intensity values of each channel in the RGB color space of a biopsy, the transport solution, and the background of a single frame (Table S1). This comparison revealed that $(\boldsymbol{R}-\boldsymbol{G})+(\boldsymbol{R}-\boldsymbol{B})$ is the most significant distinguishing value between the three components. After performing this channel manipulation, the image was transferred into a binary image using MATLAB's imbinarize() function with a threshold of 0.47. ${ }^{17}$ After binarizing, the image was morphologically opened using MATLAB's imopen() function to decrease the noise and make regions more distinct. Small fragments that are irrelevant to successful CoreView function may exist and create identifiable regions in this step. These regions, which are smaller than 1000 sq. pixels, are simply removed after this step to prevent tracking. A green rectangle was generated onto the frame around the identified biopsy using a bounding box and centroid determined using MATLAB's regionprops() function.

Multiple object tracking using tracks (video tracking). Some tissues may undergo significant fragmentation during removal and transport. Furthermore, air may be present in the needle, creating bubbles upon biopsy removal. To account for the tracking and indexing of multiple objects at once, each detected object was assigned a track that is maintained between frames. Each track was assigned the area, bounding box, and centroid properties of the distributed region using MATLAB's regionprops() function. Before tracks were assigned or modified, detections are made by the algorithm using a tracking function that finds the difference between the current frame and the last frame and applies the previously described frame tracking algorithm. To determine which track detections were being assigned to, we employed an area difference matrix defined as:

$$
A D_{i, j}=\mid \text { TrackArea }_{i}-\text { DetectionArea }_{j} \mid
$$

where $i$ is the index of tracks and $j$ is the index of detections. The track with the least area difference, identified by the above matrix, was used to assign detections (Fig. S2). If more tracks were created in a previous frame than detections that existed in the current frame, i.e., the fragments were compressed together, at least one track must be deleted for simplicity. The same area difference matrix track assignment method is used, but the track(s) with the largest area difference is/are removed.

In the RGB space, the tracking algorithm cannot reliably distinguish bubbles from the background. It is important to identify any bubbles that exist in the chip because they add compliance to the fluidic system, preventing optimal plug flow performance, and can also be indistinguishable from tissue by photosensors. In order to detect bubbles, the frame must be transferred into the HSV color space. While the background varies in this space due to location and/or lighting, a single bubble does not (Table S2). With this improvement, the same frame tracking and video tracking algorithms were used to detect any tissue, in the RGB color space, and gas bubbles, in the HSV color space, in the chip. To distinguish from the biopsy, bubbles were marked by solid red circles instead of green bounding boxes. A flow chart of the full biopsy and bubble tracking algorithm can be seen in Fig. S3.



Fig. 6 CAD cross-section of the 3D printed biopsy segmentation proof-of-concept device colored and labeled to show individual parts. The device uses hydraulic cylinders (D, F) to vertically and horizontally move a blade (J) across the channel (C). Other components include the housing box (A), fluidic chip (B), supplemental fluid port $(E)$, pistons $(G-H)$, and blade holder (I)

\section{Biopsy segmentation proof-of-concept device}

On-chip biopsy segmentation would be a valuable addition to the CoreView cartridge because it enables multiple downstream diagnostic tests from one biopsy and allows for the sampling of a single identified tumorous selection from a CNB. To perform such a task, we require a module that could repeatedly target and cleanly cut a specific location along the biopsy. To achieve this operation, we designed for minimal fluid flow around the biopsy to prevent motion, a sharp cutting device, and a clear optical window to monitor segmentation. A proof-of-concept biopsy segmentation device was 3D printed, as described previously, to test various blade forces and trajectories as well as cutting channel geometries (Fig. 6). The device 
used conventional rigid hydraulic cylinder design practices and allowed the transport channel section to be interchanged to test variations of the channel-blade interface without printing multiple iterations.

Assembly of components. The biopsy segmentation proof-ofconcept device consisted of many 3D printed parts, all finish machined to tight tolerances $( \pm 12 \mu \mathrm{m}$ ) with micro-grain carbide tooling on a manual lathe. The first component of the device was a housing box (Fig. 6A) sealed onto a fluidic chip (B) with a cutting chamber (C) using and a silicone gasket. The opposite side of the housing box was sealed onto a vertical actuating cylinder component (D) in the same manner. This component also acted as a top cover and contained a supplemental fluid port (E) for fluid displaced during actuation and air bubble purging. Both the housing box and the vertical cylinder cover were built with guides to constrain the motion of the pistons to a single direction. The horizontal cylinder (F) threaded into the right side of the housing box to allow for the installment of the internal moving components and was sealed with a dash 013 square profile O-ring. Each actuator contained its own piston (G-H), sealed with dash 010 double X-profile O-rings fitted to precision machined grooves in the pistons, that were designed with slots to guide the blade holder (I) via stainless dowel pins. This guided movement should move the stainless-steel \#23 scalpel blade (J), which is adhered to the blade holder with epoxy. The blade was intended to have a high ratio of horizontal sliding to vertical descending movement. During operation, the entire device was filled with PBS.

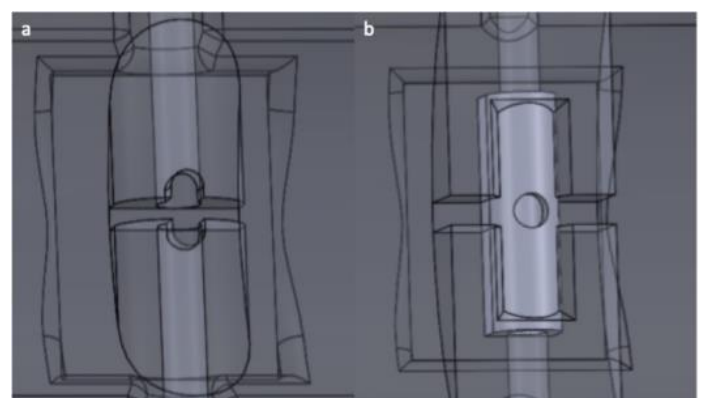

Fig. 7 CAD image of the (a) slotted cutting chamber and (b) elastomeric buffer cutting chamber of the 3D printed biopsy segmentation proofof-concept device

Testing the biopsy segmentation device. Two different cutting chambers were designed and tested (Fig. 7). The first and most simple design was a slotted cutting chamber, which had a horizontal gap in the channel to house the blade and a circular cut-out for postsegmentation biopsy separation via fluid flow. The second design was an elastomeric buffer cutting chamber, which had a similar horizontal gap and circular cut-out, but a large portion of the channel was open for the placement of a 3D printed elastomeric buffer. This buffer was very elastic, allowing the cut to close up after the blade retracts. Both designs were tested for successful biopsy segmentation and separation using biopsies from a 14-gauge, 22$\mathrm{mm}$-long CNB gun (MaxCore, Bard Biopsy). Both designs were connected to the CoreView millifluidics system via the transport pump and stain waste valves. They were also connected to the biopsy removal proof-of-concept device for easy biopsy removal and input into the tubing. The slotted cutting chamber device was tested with 6 porcine kidney CNBs and relied on manual control of the hydraulic cylinders via PBS-filled syringes. The needle was manipulated vertically and horizontally until the biopsy was successfully cut, and observations were recorded. The elastomeric buffer cutting chamber device was tested with CNBs of fresh porcine breast $(\mathrm{N}=3)$, kidney $(\mathrm{N}=7)$, and pancreas $(\mathrm{N}=6)$ tissue. These tissues have very different mechanical properties, which enable the assessment of the robustness of the proof-of-concept device ${ }^{8-9}$. Similar methods were used to manipulate the blade, and the number of passes along with any observations were recorded.

\section{Results and discussion}

Our histopathology lab on a chip millifluidic system, named CoreView, is centered around a clear cartridge that allows for the self-containment and automation of all biopsy handling steps of histopathology (Fig. 3). This cartridge is designed to be massproduced by injection molding but is currently 3D printed as single prototypes. The CoreView chip is also designed to be disposable but can be sterilized or ultra-cleaned with high-level disinfection processes used for endoscope reprocessing. Millifluidics is preferred to microfluidics for histopathology because it preserves tissue architecture and allows for optical imaging of both diseased cells and their microenvironment. A schematic of the entire millifluidic system prototype can be seen in Fig. 2. We have demonstrated that our CoreView chip successfully uses millifluidics to remove, transport, stain, image, and park needle biopsies (Movie S1).

\begin{tabular}{|c|c|c|c|c|c|c|}
\hline \multirow{2}{*}{ Tissue } & \multirow{2}{*}{ N } & Removed & Biopsy & \multicolumn{3}{|c|}{ Removal Pressure (psi) } \\
\cline { 5 - 7 } & & from Needle & Stayed Intact & Mean & Median & Std. Dev. \\
\hline Breast & 29 & $89.66 \%$ & $75.86 \%$ & 7.07 & 3.5 & 7.216 \\
\hline Kidney & 21 & $100.00 \%$ & $76.19 \%$ & 3.38 & 2.0 & 5.035 \\
\hline Liver & 40 & $97.50 \%$ & $67.50 \%$ & 4.00 & 2.0 & 4.114 \\
\hline Lung & 25 & $84.00 \%$ & $96.00 \%$ & 9.00 & 10.0 & 7.826 \\
\hline Lymph Node & 31 & $96.77 \%$ & $77.42 \%$ & 4.43 & 2.0 & 3.770 \\
\hline
\end{tabular}

Table 1 Biopsy removal efficiency using the 3D printed biopsy removal proof-of-concept module containing 14-g side-cut needle biopsies.

\section{Biopsy removal from its needle device}

Results from the biopsy removal experiments are presented in Tables 1-3 and S3-S4. These results show that successful biopsy removal using the proof-of-concept device and the integrated chip module is efficient and reproducible across a wide range of tissues. The first discrepancy is the percent of biopsies that were removed from the needle. In the proof-of-concept module experiment, most CNBs were fully released, but only $84 \%$ of lung CNBs were removed while $100 \%$ were removed in the integrated cartridge (Table 1-2). The most likely reason for this difference, and differences in other organs, is the freshness of the sample. Fresh tissue is found to change material properties within hours of resection ${ }^{18}$. In the proof-of-concept module experiments, the tissue was either fresh (used within five hours of resection) or refrigerated (for 19+ hours after resection), while the integrated chip experiments only used fresh tissue. The differences in adhesion to surfaces ("stickiness"), elasticity, and the tendency to break into fragments are very pronounced between these post-resection times. As a result, the ability of a 
CNB to be removed and stay intact is highly dependent on time since resection (time ex vivo).

\begin{tabular}{|c|c|c|c|c|c|}
\hline \multirow{2}{*}{ Tissue } & Removed & \multirow{2}{*}{$\begin{array}{c}\text { Biopsy } \\
\text { from Needle }\end{array}$} & \multicolumn{3}{|c|}{ Removal Pressure (psi) } \\
\cline { 4 - 6 } & & Stayed Intact & Mean & Median & Std. Dev. \\
\hline Breast & $100 \%$ & $90 \%$ & 3.50 & 3.0 & 1.933 \\
\hline Kidney & $100 \%$ & $100 \%$ & 2.40 & 2.0 & 0.821 \\
\hline Lung & $100 \%$ & $95 \%$ & 3.10 & 3.0 & 1.210 \\
\hline Pancreas & $100 \%$ & $94 \%$ & 3.50 & 2.0 & 2.328 \\
\hline
\end{tabular}

Table 2 Biopsy removal efficiency in the integrated CoreView chip using 14-g side-cut needle biopsies.

The experiments assessing the range of side-cut needle sizes showed that the biopsy removal module can remove all CNBs 14-18-gauge with low pressure flow (Table 3). This preliminary data indicates that higher gauge (smaller diameter) needles may require a higher driving pressure to remove the biopsy, but the sample size is too small to draw significant conclusions. The biopsy removal module can also be used to pull a CNB from endcut needles with assistance from a central wire or stylet running out through the needle tip during fluidic flow. Minor channelsize modifications will be needed to accept needles carrying larger cores, such as 9-12-gauge used for larger breast tumors.

\begin{tabular}{|c|c|c|c|c|c|c|}
\hline \multirow{2}{*}{$\begin{array}{c}\text { Needle } \\
\text { Gauge }\end{array}$} & $\mathrm{N}$ & \multirow{2}{*}{$\begin{array}{c}\text { Removed } \\
\text { from Needle }\end{array}$} & Biopsy Stayed & \multicolumn{3}{|c|}{ PSI } \\
\cline { 5 - 7 } & & Intact & Mean & Median & St Dev \\
\hline 14 & 10 & $100 \%$ & $100 \%$ & 3.60 & 3.00 & 2.07 \\
\hline 16 & 10 & $100 \%$ & $100 \%$ & 3.20 & 3.00 & 1.40 \\
\hline 18 & 10 & $100 \%$ & $80 \%$ & 5.40 & 5.00 & 2.67 \\
\hline
\end{tabular}

Table 3 Biopsy removal efficiency of various side-cutting needle sizes in the integrated CoreView chip. All CNBs were resected from porcine kidney refrigerated for 21 days.

The integrated cartridge biopsy removal experiments show that the bottle pressure of the high-flow PBS can be lower than deemed necessary by the concept module experiments. Utilizing this result to modify the Hi-flow PBS system will prevent excessive PBS waste and help to reduce a CNB "overshoot" of the staining/imaging compartment if it is released in the first couple pulses. Although these results are very promising, it was still found that around $5 \%$ of CNBs will be broken during removal, depending on the quality and composition of the biopsy. Fragmentation can cause challenges in biopsy tracking and make it harder to accumulate all tissue in the imaging chamber. To overcome these challenges, a new module containing an in-line sifter or sieve can be used to amass all the fragments into a smaller volume which can be transported back into the imaging chamber as a single packet.

\section{Biopsy transport, staining, and imaging}

We have shown that the CoreView millifluidic histopathology on a chip can successfully and reliably transport, stain, and image small needle biopsies. Furthermore, we have demonstrated that reliable bidirectional fluid transport can be achieved with 0.5-mm positioning accuracy. Full operation of two current prototypes can be seen in Movie S1. The first prototype chip used in the video (Fig. 3) shows successful removal, transport, and parking of a fresh porcine kidney CNB using system millifluidics. This biopsy is imaged using oblique reflectance for the computer-aided detection of low-contrast glomeruli found in diseased or transplanted kidney tissue (Fig. 5). The image processing of the CNB provides higher contrast than typical white-light imaging used in a pathologist's portable inspection scope. Furthermore, assuming remote pathologists can image and confirm the glomeruli count in each CNB within 4 minutes, this form of tele-pathology could save pathologists at least 30 minutes of travel time.

The second prototype chip used in the video shows bidirectional transport and staining of a fresh porcine breast CNB using CoreView millifluidics. Interestingly, during the sped up, 1minute staining and rinsing section of Movie S1, a large air bubble was introduced with the staining solution that adhered to the biopsy, adding compliance and surface tension. This addition resulted in atypical movement of the biopsy during the slower staining and rinsing pulsatile flows. By priming the system prior to needle insertion, these anomalies appear to be prevented. After staining and rinsing, imaging of breast nuclei, stained blue on the surface of the CNB, was demonstrated using MUSE. This new method of non-slide-based histology is being developed for the rapid assessment of tumor-rich regions in breast tissue with improvements in image quality needed for high-quality diagnostic applications. ${ }^{19}$ Although it is still not a commercially available diagnostic technique, MUSE is a good choice for the ROSE adequacy capabilities of our system. Images produced using MUSE, such as the ones displayed in this section of the video, can be used to identify tumor-dense regions in CNBs.

Although Movie S1 shows the successful demonstration of a nearly completed CoreView chip and millifluidic system, the device is still not complete. Firstly, no system-wide fullautomation has been developed, with the exception of the LABVIEW interface that controls and monitors all pumps, valves, and sensors. As a result, each millifluidic pulse, or series of pulses, is controlled by the operator. Multiple systems have been developed, or are still under development, to enable CoreView automation, including the real-time biopsy tracking algorithm and a linear lens-phototransistor object detection system. The final chip will likely feature a linear lens and phototransistor after the biopsy removal module to turn off the high-flow pump more quickly than the biopsy tracking algorithm can. This feature will prevent any biopsy overshoot of the staining chamber.

\section{Biopsy tracking}

The complete biopsy tracking algorithm successfully identified and tagged all biopsies, biopsy fragments, and bubbles on all videos. The detection of a biopsy in the RGB color space is shown in Fig. $\mathbf{S 4}$ and the detection of an air bubble in the HSV color space is shown in Fig. S5. The final result of biopsy and bubble tracking is shown in Fig. 8 and Movie S2. Different illumination conditions were also used in many of the videos, but no change in tracking performance was perceived. This is an 
important quality for the clinical application of the CoreView system, especially in the developing world, where the point-ofcare may not be in a hospital/clinic. A robust biopsy tracking algorithm is necessary for the automation of the CoreView system because no two needle biopsies have the exact same properties. Different biopsies are not removed nor transported at the same rates, which means that a set, finite number of pulses for transport from the needle to the staining and imaging chamber cannot exist. In the future, our biopsy tracking algorithm will enable LabVIEW to close the control-loop for advancing the process sequence to precisely stop the biopsy transport at each module.

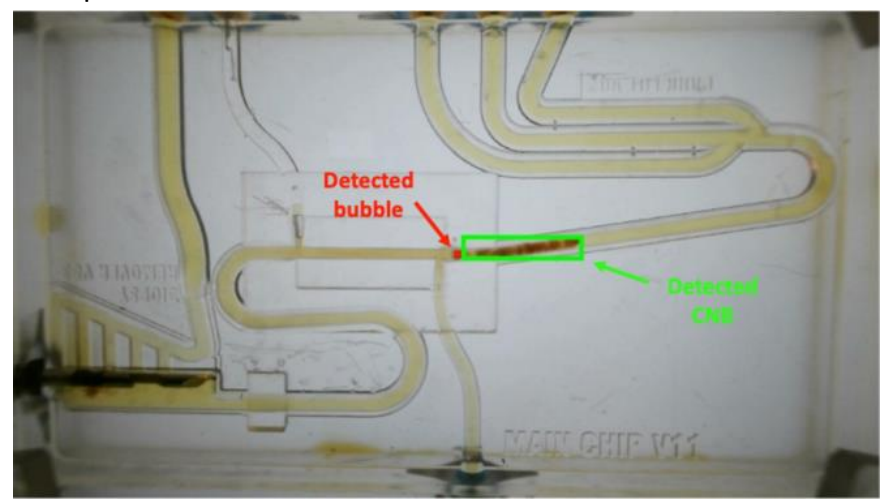

Fig. 8 Result of the biopsy and gas bubble detection algorithm. This image is of one frame of a video showing the transport of a 14-g, 20-mm-long porcine kidney CNB (green box) and a lone bubble (red circle).

While this algorithm is intended to be used for real-time tracking to guide system automation, its current form is limited by a speed of around 9-10 frames-per-second (fps) while the videos used had a frame rate of $25 \mathrm{fps}$. This limitation may be remedied by resizing each frame into a lower resolution image, increasing computational resources, or reprogramming into more rapidly executable algorithms. Additionally, the algorithm speed can be greatly improved by only detecting biopsies and bubbles in the CoreView chip fluidic channels, but current prototyping makes the immediate implementation of this change infeasible.

\section{Biopsy segmentation}

Biopsy segmentation was performed as shown in Fig. 9. Both proof-of-concept biopsy segmentation devices were able to successfully cut the tested CNBs, but each device had its own limitations.

The slotted cutting chamber device showed consistent cutting performance after multiple passes across the biopsies with minimal tissue snagging during transport due to its relatively simple geometry. Multiple passes were required to produce cutting forces high enough to completely cut the biopsies yet low enough to avoid pulling the tissue into the device through the cut-out. The tendency to inadvertently pull biopsies out of the channel could be remedied by decreasing the clearance between the blade and the cut-out or by adding a moving component, such as an elastomeric buffer, at the cost of manufacturing complexity, to provide a zero-clearance fit.
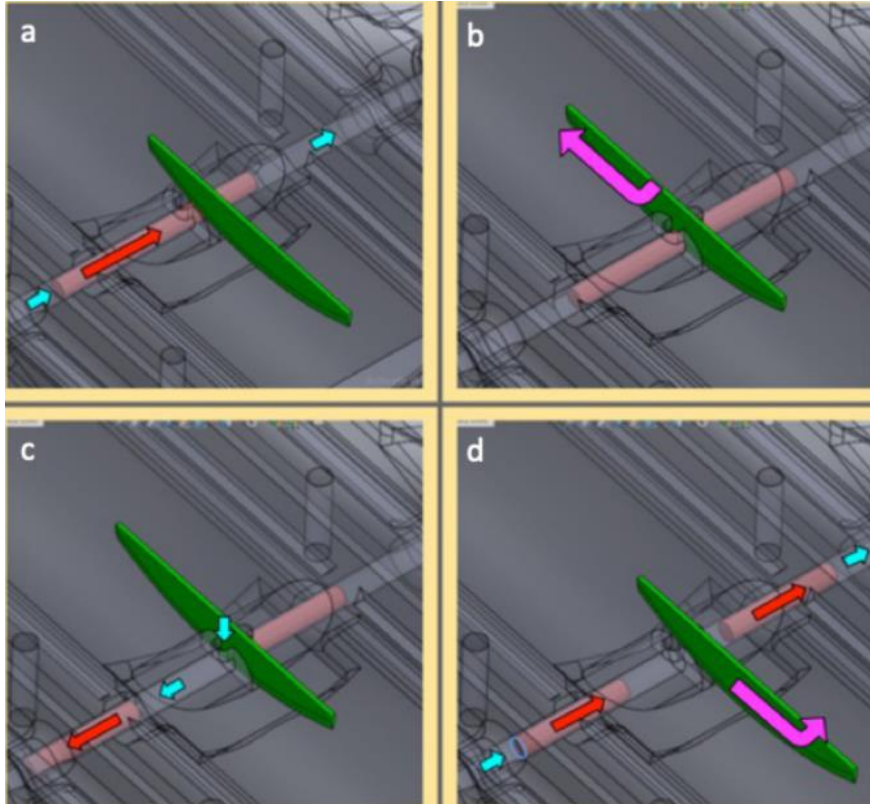

Fig. 9 A series of CAD images showing the intended blade movements of the biopsy segmentation proof-of-concept device. (a) Placement of the CNB (blush red) using transport millifluidics prior to blade (green) movement. (b) Slicing (pink) with the blade. (c) Pushing the biopsy away from the blade using inflow (cyan) through the circular cutout. (d) Blade retraction and biopsy transport.

The elastomeric buffer cutting chamber device houses the biopsy in an elastic sheath that is cut with the biopsy. This design constrained the lateral motion of the biopsy near the blade more efficiently than the slotted cutting chamber and allowed for the higher cutting forces needed to cut the tissue in one or two passes (Fig. S6). However, the softer and more fibrous breast biopsies were frequently snagged between the buffer and the channel, prior to entering the buffer, and at the buffer's pinch point after the blade was retracted.

Overall, these experiments prove that successful biopsy segmentation using fluidics is possible and has the potential to be integrated into a CoreView chip. However, these proof-ofconcept devices are over-sized and inordinately complex. The final module will likely employ fewer, simpler, and less costly parts with compliant devices such as diaphragms, bending actuators using differential stiffness, or extending bellows. ${ }^{20} \mathrm{~A}$ channel design that allows the biopsy to be cut with simple 1D motion will further simplify this mechanism. Exact components will be determined with future iterations and experiments.

\section{Implications for the future of pathology}

CoreView demonstrates the potential to solve many current and future problems associated with small needle biopsy histopathology. Firstly, the ability to use millifluidics to automatically remove biopsies from their needle, reliably transport, stain, image, store, and segment biopsies can alleviate many problems associated with manually handling biohazardous human tissue specimens and their stains and fixatives. Automating biopsy handling also allows for non- 
destructive tissue preservation from needle procurement to imaging and later fixation. These abilities are a major improvement in the field of ROSE technologies and is what will distinguish CoreView from TP and other more destructive tests. Secondly, using fresh tissue enables the sample preparation of several downstream diagnostic tests, including RNA integrity number (RIN) for gene expression analysis, and other '-omics' analyses. Fixation disrupts DNA, RNA, and other sensitive molecules, preventing valuable '-omics' analysis that can guide personalized cancer therapies. ${ }^{21}$ Using the segmentation module to divide needle biopsies and the parking module to hold each segment separately, allows for tissue fixation and/or preservation with different solutions. For the renal CNB example, biopsy segments would be deposited in formalin for conventional histopathology, chemical preservative for electron microscopy, and PBS for future immunofluorescence imaging. CoreView also has the potential to monitor tissue fixation using a novel non-contact module that has been developed to aid in the automation of the system. This 3Dprinted low-cost monitor measures the optical transmittance of a CNB in a millifluidic chamber and can be used to determine when the biopsy is optimally formalin-fixed.22 In the future, additional microfluidic modules can be added to the CoreView millifluidic chip for maintaining vitality of fresh tissue for realtime chemical monitoring, reactions to applied chemotherapy drugs, and analysis of extracted analytes from CNBs. ${ }^{23-25}$ The ability of CoreView to produce histopathologic images in minutes instead of hours to days presents a major global health solution. Breast cancer is the most common cancer and secondleading cause of cancer-related deaths in sub-Saharan Africa. ${ }^{26}$ Standard CNB histopathology requires specialized equipment and laboratories present in less than $10 \%$ of hospitals in lowincome countries. ${ }^{27}$ Additionally, skilled personnel are also scarce in these regions, best exemplified by Africa's 100 -fold fewer pathologists-per-capita than the US and Canada. ${ }^{27-28} \mathrm{~A}$ lower-cost and portable CoreView system using MUSE or other emerging thick-tissue imaging techniques can be used to provide same-day therapeutic decision making in settings where follow-up appointments for affected patients may be unattainable. ${ }^{29}$

\section{Conclusions}

In this paper, we present the current state of CoreView, our goa of creating a millifluidic histopathology lab on a chip device that handles fresh small needle biopsies from resection to imaging. We demonstrate that pulsatile millifluidics can be used to reliably remove CNBs from their side-cut needles (14-18-gauge), transport CNBs through a 2-mm channel with $0.5-\mathrm{mm}$ positioning accuracy, and stain and rinse stationary CNBs. We also confirm that fluidics can be used to cut fresh CNBs into multiple segments for separate downstream diagnostics. Lastly, we show that a real-time biopsy tracking algorithm can be employed to enable system automation using machine vision.
With the current low-resolution optical microscopy in this initial report, the CoreView system is able to perform ROSE adequacy within a few minutes after biopsy acquisition to ensure the proper location was resected. This process is very important for organs such as the kidney, where each biopsy has a finite risk of causing a major adverse event, as well as for applications in global health. In addition, our novel approach of counting glomeruli eliminates the need for the pathologist's presence in the interventional radiology suite, saving precious time and personal protective equipment.

\section{Conflicts of interest}

Most authors participate in the royalty sharing program at the University of Washington, which owns CoreView patent filings. EJS is also a shareholder in ROSEbiopsy Inc., which also owns some CoreView patents.

\section{Acknowledgements}

Funding provided by the University of Washington CoMotion Innovation Fund, the Washington Research Foundation (Phase 1 Award and Undergraduate Research Fellowship), and the National Cancer Institute Exploratory R21 CA 246359 award. We thank Shawn Swanson for loaning us his personal Form 3 printer. Yak-Nam Wang from UW Professor Mike Bailey's team for providing fresh discarded pig tissues. We thank Dr. Rodney Schmidt, MD, for his initial mentorship of CoreView from a pathologist's perspective. Lastly, we thank Dr. Richard Levenson, MD, for his insight on slide-free thick tissue histological imaging.

\section{Notes and references}

1 M. Titford, Journal of Histotechnology, 2006, 29, 99.

2 K.P.H. Pritzker and H.J. Nieminen, Arch Pathol Lab Med., 2019, 143, 1399.

3 P.A. VanderLaan, Cancer, 2016, 124, 862.

4 S. Satturwar, N. Rekhtman, O. Lin and L. Pantanowitz, Cytopathology, 2020, 9, 322.

5 K.A. Shaikh, K.S. Ryu, E.D. Goluch, J. Nam, J. Liu, C.S. Thaxton, T.N. Chiesl, A.E. Barron, Y. Lu, C.A. Mirkin and C. Liu, PNAS, 2005, 102, 9745.

6 K.C. Bhargava, B. Thompson and N. Malmstadt, PNAS, 2014, $111,15013$.

7 S. You, Y. Sun, E.J. Chaney, Y. Zhao, J. Chen, S.A. Boppart and H. Tu, Biomed Opt Express, 2018, 9, 5240.

8 J. Rosen, J.D. Brown, S. De, M. Sinanan and B. Hannaford, Journal of biomechanical engineering, 2008, 130, 021020.

9 A.W. Hudnut, B. Babaei, S. Liu, B.K. Larson, S.M. Mumenthaler and A.M. Armani, Biomedical optics express 2017, 8, 4663 .

10 J.F. Hale, D.A. McDonald and J.R. Womersley, J. Physiology, $1955,128,629$

11 S. Tsangaris and N. Stergiopulos, J. Biomechanics, 1988, 21 263.

12 O. San and A. Staples, Journal of Mechanics in Medicine and Biology, 2012, 12, 1250052-1. 
13 K. Zuiderveld, Graphic Gems IV, Academic Press Professional, San Diego, 1994.

14 H. Bay, T. Tuytelaars and L. Van Gool, SURF: Speeded Up Robust Features, Springer, Berlin, Heidelberg, 2006.

15 M.A. Fischler and R.C. Bolles, Random sample consensus: $A$ paradigm for model fitting with applications to image analysis and automated cartography, Communications of the ACM, 1981.

16 F. Fereidouni, Z. T. Harmany, M. Tian, A. Todd, J. A. Kintner, J. D. McPherson, A. D. Borowsky, J. Bishop, M. Lechpammer, S. G. Demos and R. Levenson, Nature Biomedical Engineering, 2017, 1, 957.

17 MathWorks Help Center, https://www.mathworks.com/help/index.html, (Apr. 20, 2021).

18 H. Nitta, B.D. Kelly, C. Allred, S. Jewell, P. Banks, E. Dennis and T.M. Grogan, Pathology International, 2016, 66, 313.

19 W. Xie, Y. Chen, Y. Wang, L. Wei, C. Yin, A. Glaser, M. Fauver, E. Seibel, S. Dintzis, J. Vaughan, N. Reder and J. Liu, Journal of Biomedical Optics, 2019, 24, 026501.

20 K. Ogura, S. Wakimoto, K. Suzumori and Y. Nishioka, Micro pneumatic curling actuator - Nematode actuator, IEEE International Conference on Robotics and Biomimetics, 2008.

21 A. Frankel, ANZ journal of surgery, 2012, 82, 395.

22 S. Lim, PhD thesis, University of Washington, 2020.

23 L.T. Cheah, Y.H. Dou, A.M.L. Seymour, C.E. Dyer, S.J. Haswell, J.D. Wadhawan and J. Greenman, Lab on a Chip, 2010, 10, 2720.

24 L.F. Horowitz, A.D. Rodriguez, T. Ray and A. Folch, Microsystems \& Nanoengineering, 2020, 6.

25 S. Abdulwahab, A.H.C. Ng, M.D. Chamberlain, H. Ahmado, L.A. Behan, H. Gomaa, R.F. Casper and A.R. Wheeler, Lab on a Chip, 2017, 17, 1594.

26 The Cancer Atlas, https://canceratlas.cancer.org/theburden/sub-saharan-africa/, (May 23, 2021).

27 African Strategies for Advancing Pathology, http://www.pathologyinafrica.org/surveydata/mapdata.php?type=n_path\%7Cminmax\%7C1\%7C1\%7C1\%7CNumber+of+Pathologists+Per+Milli on+Population\#, (May 23, 2021).

28 D.M. Metter, T.J. Colgan, S.T. Leung, C.F. Timmons and J.Y. Park, JAMA Network Open, 2019, 2, e194337.

29 R. Levenson, F. Fereidouni and T. Morningstar, Laboratory Investigation, 2021, 101. 